\title{
Early estimation of work contents for planning the one-of-a-kind production by the example of shipbuilding
}

\author{
Jan Niklas Sikorra, Axel Friedewald and Hermann Lödding \\ Hamburg University of Technology, Institute of Production Management and Technology, 21073 Hamburg, Germany
}

\begin{abstract}
A transparent, reliable early estimation of work contents is crucial for capacity planning in shipyards. Yet most of the shipyards rely on practical experience of specialized production planners, for the early capacity estimation, which are prone to errors, as a bill of material is not available in the early stages of production planning in the one-ofa-kind production. This is due to a yet unfinished construction of the ship. This paper will discuss a method for creating a computer-supported, transparent, more reliable way for the estimation of work content, at an early planning stage of a one-of-a-kind production, using a generated bill of material. The bill of material is generated automatically using parametrized templates and contains steel and outfitting parts. Therefore a tool has been developed that generates steel and outfitting parts from parametrized templates. From the bill-of-material, the work content in hours is estimated by linking the parts in the bill-of-material to a process. The operation time can then be calculated by dividing the work content by the capacity of the work system. The operation time can then be used for scheduling the one-of-a-kind production.
\end{abstract}

\section{Introduction}

Work content estimation in the early phase of planning the one-of-a-kind production of complex products is uncertain and prone to inconsistencies. Yet decisions made in the early phase of production planning in the one-of-a-kind production have a high impact on delivering on time. Furthermore as the price of the product is fixed, costs have to be estimated, which makes wrong estimations costly. Therefore generating better information for production planning in the one-of-a-kind production is a necessity to gain an advantage against competitors on the world market.

The uncertainty in the early phase of planning the one-of-a-kind production origins from a lack of design data, as design, production planning and work scheduling have to be done in parallel, to minimize the throughput time of a one-of-a-kind product.

The paper discusses an approach on how to estimate the work content in the early phase of planning the oneof-a-kind production by coping with the lack of design data, by the example of shipbuilding. It therefore describes a way of generating a bill of material (BOM) from past data, using parameterized templates. It also introduces a parameter based estimation for calculating the work content from the part data in the BOM. The work content can then be used for creating more realistic schedules and for estimating the capacity demand.

\section{State of the Art}

One-of-a-kind production means, that a product is only produced once. In the one-of-a-kind production of complex goods, like a building, a plant or a ship, design and planning are often done in parallel, which causes a lack of data in the early phase of planning a one-of-a-kind product [1-3].

In the early phase of planning the one-of-a-kind production and scheduling the work, little design information of the one-of-a-kind product is available, as the planning is done in parallel to the design, to reduce the throughput time of the whole project. Due to this a target conflict arises between having design data available to enable a certain planning and having a low throughput time for the whole project and low costs in total [4].

If all parts of a product, as well as the parts hierarchy were known and documented in the BOM and the processing times, lot sizes and set up times were known, the work content in hours could be calculated [5]. From the work content, the operation time in days can be derives, which is needed to schedule the production.

Yet without the design, the exact work steps are not known yet. Therefore calculating the work content has to base on vague estimations and old ships data. While there are the DIN 277 and DIN 276 for calculating costs of constructions, and a method to do so, such a method does not exist in shipbuilding [6]. There also are approaches of estimating cost for plants, whereas the parts are known 
for the cost estimation the methods either work bottom up or on each level of the design hierarchy [7-9].

There is the necessity to guess a BOM at the early stage of planning a one-of-a kind production. Shipyards currently reuse old design data, but as the ships change tremendously, the approach of just reusing data does not deliver sufficient results [4].

First approaches to develop a tool to generate simple part data have been made in the research project GeneSim, in a different context. The idea in GeneSim was to fill gaps in simulation databases by generating parts. For this purpose a very simple software to create basic structural steel parts (profiles and plates) was developed [10]. The software is not yet able to create a sufficient BOM.

As a result the question is raised whether a BOM can be guessed at the early stage of planning. A sufficiently good BOM could on the one hand close the gap that the unfinished design creates in the early stage of planning the one-of-a-kind production, on the other hand it could create a new step in today's typical planning of the oneof-a-kind production which would lead to a higher transparency.

\section{Planning workflow for work content estimation}

Estimating a bill of material while planning the oneof-a-kind production delivers two advantages. The first advantage of estimating a BOM is a higher transparency in the planning process, as product uncertainties and process uncertainties are separated. This enables a profound controlling and identification of errors in the planning process is possible, which enables the planners to learn in a systematic way from their mistakes. The second advantage is, that a sufficiently good estimation of the BOM can improve the certainty of the planning.

This paper describes a new approach for planning the one-of-the-kind production (Figure 1).

It differs from traditional approaches by taking into account an estimated BOM. The BOM fills a knowledge gap in planning the one-of-a-kind production, by estimating important parts, which are not yet constructed. The approach as shown in figure 1 consists of eight steps. The first four steps deal with estimating parameters for the generation of important parts for the BOM. Steps five to seven deal with generating the BOM. Step eight describes the estimation of the work content. The work content can then be used for cost estimation and production scheduling.

The following paragraphs will describe each step of the ne planning workflow estimating a BOM in the oneof-a-kind production.

\subsection{Product specifications}

Already when negotiating a contract, first product specifications are set. Latest by closing the contract, all product specifications for the one-of-a-kind product are fixed. These product specification are the base for the planning approach described in this paper and should be used as early as they are available.
An example for a product specification in the shipbuilding industry would be the ice class. Knowing the ice class, product specifications can be used for calculating global and local parameters.

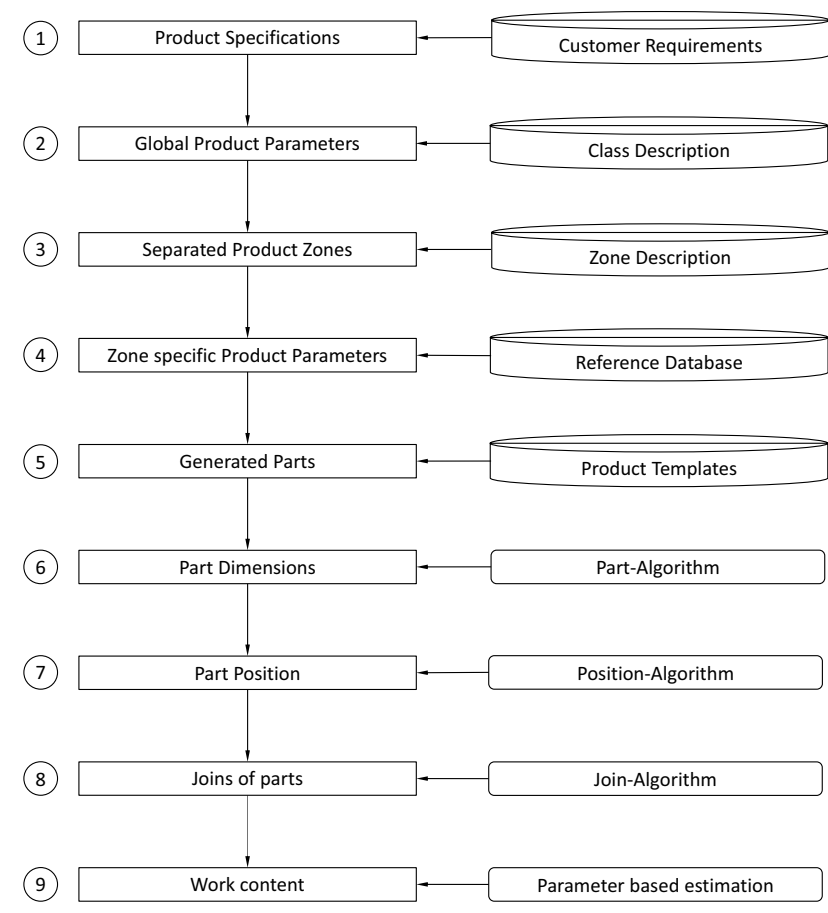

Figure 1. New approach for planning the one-of-a-kind production

\subsection{Global product parameters}

Global product parameters are derived from the ships specifications. To differentiate global and local parameters minimizes the work content for the planner, as global parameters are only derived once for a ship. The parameters are needed for the calculation of the BOM, as well as the local parameters. Global parameters can either be derived from guidelines, from former projects or calculations. For example the ship specification ice class would define a global parameter, the thickness of the hull.

\subsection{Separated product zones}

To create clusters with similarities in the design, the ship is divided into zones. While different zones might differ a lot, a zone is assumed to bare a lot of similarities. This allows the estimation of zone specific parameters which describe a zone relatively precisely. Therefore after global parameters are set, zones will be defined, which enclose an area with a specific function, for example the engine room of a ship, or the double bottom. If parameters were derived for areas with strongly differing functionalities, the variance of parameters in this zone might be too big and therefore the results of the estimation based on the parameters would be uncertain.

\subsection{Zone specific product parameters}


Now that the one-of-a-kind product is divided into zones, zone specific parameters can be derived. Zone specific parameters are used to describe specifications that cannot be described on a global level with enough certainty. The parameters can once again be derived from directives, old projects or calculations. On a Ferry for example the stiffener spacing, width and height on the car deck can be derived from the expected load.

\subsection{Generated parts}

The calculating of the parts and their hierarchy starts at step five of figure 1. The goal of calculating parts and hierarchy is to create a BOM that reflects the new build one-of-a-kind product in the best way possible and can be used for work content estimation.

To minimize the planning efforts, the BOM is generated using templates. The templates can be parametrized using the global and local parameters derived in step one to four. The templates are structured hierarchic to reflect the structures of the designs, which's data they inhabit. The result is a hierarchic list of the parts, of the estimated design. For example a design panel (figure 2) can be created as a hierarchy consisting of the panel on the top level and below this plates and profiles.

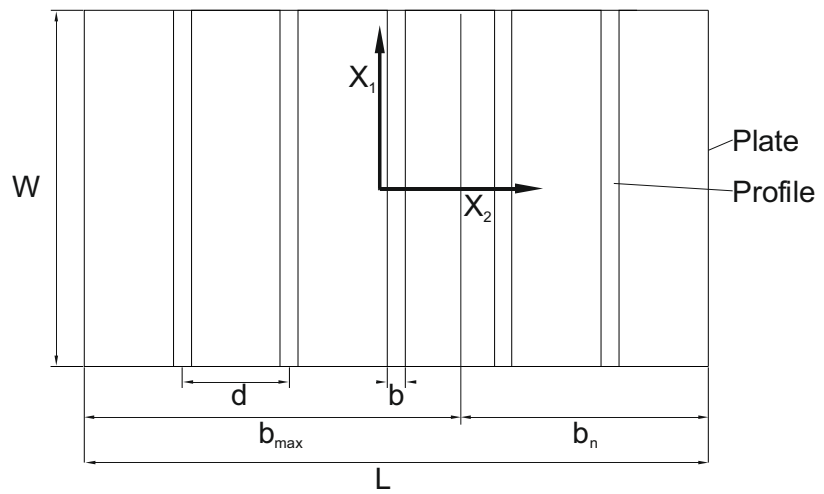

Figure 2. Parametrized construction (panel)

A complete BOM must inhabit all parts of the design. If a part occurs several times, all of the reoccurrences have to be calculated. Therefore the number of profiles ( $\left.n_{\text {profiles }}\right)$ in the construction in figure 2 can be derived by rounding down the length of the construction $(L)$ divided by the distance of the stiffeners $(d)$ according to equation 1 .

$$
n_{\text {profiles }}=\left\lfloor\frac{L}{d}\right\rfloor
$$

For a complete BOM, the reoccurrence of parts, which have a maximum size due to delivery or production constraints has to be calculated. An example for this is a plate field. In figure two, the maximum length of a plate is parameters is the parameter $b_{\max }$ in figure 2 . The parameter $b_{\max }$ specifies the maximal length of a single steel plate. After a length of $b_{\max }$, the plate has to be split. Therefore the number of single plates $(n)$, a field of plates consists of is defined by the width $(W)$ and length $(L)$ of the field of plates and the maximum width $\left(W_{\max }\right)$ and maximum length $\left(L_{\max }\right)$ of the plate. Equation 2 shows the calculation of the number of plates by the example of length. The number of plates always is an integer, therefore the results of equations one and two have to be rounded up. Therefore $n_{L}$ is the number of plates placed in the length direction.

$$
n_{L}=\left\lceil L / b_{\max }\right\rceil
$$

The same equation applies for calculations in widthdirection. $n_{W}$ therefore is the number of plates in width direction (equation 3 ).

$$
n_{W}=\left\lceil W / W_{\max }\right\rceil
$$

The total number of plates in a field of plates calculates to $n$ as stated in equation 4 .

$$
n=n_{L} \cdot n_{W}
$$

Analog to the equations stated above, reoccurring parts can be calculated and included in the hierarchy calculated for the BOM.

\subsection{Part dimensions}

For the calculation of the work content, part dimensions are important, as the work content often varies with part specific parameters. An example would be a profile, which's length would determine the length of the joins, on which it would be welded onto a plate. Part dimensions can be set in three ways. The first possibility is that they are either are defined by global or local parameters, like a maximum width, or a default width. The second possibility is that the dimensions are defined by a constraint of the construction. For example the length of a profile would equal the width of the panel in figure 2. The third possibility is that they can be calculated. For example the width of the last plate in a field of plates would be calculated to $b_{n}$ in figure 2 . To calculate the length $b_{n}$, the length of the combined $n-1$ plates before the $n$-th plate are subtracted from the length of the complete construction $L$ (equation 5).

$$
b_{n}=L-b_{\max } \cdot\left(n_{L}-1\right)
$$

\subsection{Part position}

The work content of a complex designs can depend on more parameters than the dimensions of the parts included. To calculate the work content for complex design, the position information for each part is important. For example in shipbuilding, the position information allows for the calculation of the joins of parts (step 8). This is due to three reason. The first reason is, that knowing the positon of a part, position specific parameters for calculating the work content can be introduced. The second way is a calculation of the joins in complex constructions. The intersections of the parts can differ from the length of the part. Therefore join estimation is more precise for calculating the work content than a length based parameter estimation. The position also allows part selection by defining a new sub design. The position of the part determines whether the part is in the sub design. 
The calculation of the positions is divided into two steps. First, position for the templates at the bottom of the template hierarchy are calculated using a coordinate system that is placed in the center of a construction. In the second step, if templates are combined, the coordinate systems of the sub designs are transformed into the parent design.

In step one the position of each part in this coordinate system can be specified using a vector. Reoccurring parts are placed on a line. The calculation as shown in equation 6 will be explained by the example of a profile. Equation six shows the equation for the calculation of the position of reoccurring parts where $i$ is the parts name and $\lambda$ is the index of parts.

$$
X_{i \lambda}=(\lambda-1) \cdot\left(\begin{array}{l}
x_{1} \\
x_{2} \\
x_{3}
\end{array}\right)+\left(\begin{array}{l}
c_{1} \\
c_{2} \\
c_{3}
\end{array}\right)
$$

The coordinates of the profiles in figure 2 can be calculated using equation 7 . Therefore the coordinates of the $j$-th profile can be calculated to $X_{p r, j}$.

$$
X_{p r, j}=(j-1)\left(\begin{array}{l}
0 \\
d \\
0
\end{array}\right)+\left(\begin{array}{c}
0 \\
-L / 2+d \\
0
\end{array}\right)
$$

The calculation of the position of each part of an assembly can always be calculated using vectors.

If a complex construction is created, construction templates are combined using coordinate system transformations with Euler angles. Figure 3 shows an example of a construction created with the described method.

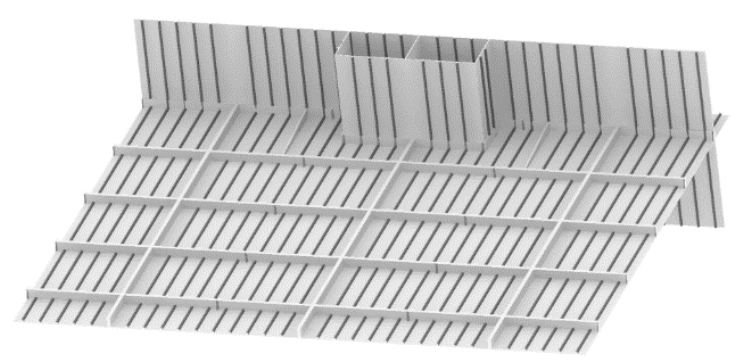

Figure 3. Generated ship's block

The advantage of the coordinate system transformation is that common assemblies can be reused and combined to create complex assemblies on a higher level in the hierarchy. Therefore the effort for describing the complex object is minimized.

\subsection{Joins of parts}

The example of shipbuilding includes a lot of steel construction. Main driver for work content when building a ship's steel hull is welding joins. Therefore a method has been developed to identify areas of contact of parts and calculate their edges and their positions. The edges are the joins of the parts. The algorithm for automatic join creation can also be used to calculate the joins of subassemblies to each other and therefore calculate the work content for different hierarchic levels of the manufacturing and assembly process in the production. This enables a faster and more reliable way of work content estimation. As the calculated joins can directly be used for parameter based work content estimation.

\subsection{Work content}

The work content $(W C)$ in hours can be calculated using equation 7 (step eight of figure 1). The equation bases on work content estimation methods. Therefore the work content for a specific part can be calculated by a part specific parameter $\left(P_{\text {Product }}\right)$, like its length, divided by its specific process speed $\left(v_{\text {Process }}\right)$ in $1 / \mathrm{min}$. The part specific parameter is introduced as the work content for the same part type might vary due to the parts volume for instance.

$$
W C_{p}=\frac{P_{\text {Product }}}{V_{\text {Process }}} \cdot \frac{1}{60}
$$

A basic table of possible parameters was created in the research conducted. For a welding the length of the join would be divided by the speed of welding. For an isolation, the work content of an isolation of a room would for example be calculated by the surface of the volume divided by the speed of isolating.

With the work content calculated, either costs for the work content of a one-of-a-kind product, in this case a ship, can be calculated, or the production can be scheduled using the estimated data.

\section{Software prototype}

The method described in this paper was implemented into a software prototype. It consists of two parts. The first part is the algorithm that enables the selection of templates and that calculates components, parts and their metadata. The second part displays the generated data and allows for its selection and manual manipulation. The software prototype is built to estimate a complete ship.

The old data is stored in templates, which can be created by hand. The templates created from old data are structured in the same hierarchy as the design. The template also specifies the parameters, the planner has to enter in order to calculate the parts, in our example the distance of stiffeners for example. Templates can be used nested, therefore one template can include another one and pass the parameters needed for the calculation to it. The software prototype then does the calculation for each of the nested templates. The nesting of the template prohibits reoccurring creation of the same assemblies. The software also contains a chooser for the templates. This tool allows the planner to visually choose a template for estimating a new assembly, which he thinks fits best 
for the future-to-build ship, which he is currently working on. The XML-Picker is shown in figure 4.

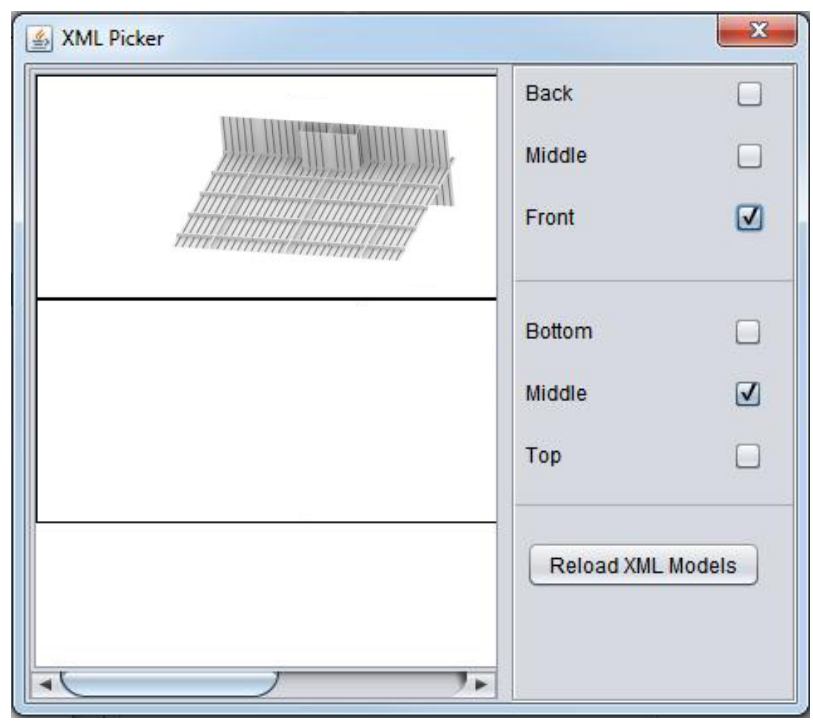

Figure 4. XML-Picker

The template is parsed by the software prototype, and the parts as well as the hierarchy are created. The parts and the hierarchy are than calculated by the first part of the software. The parts and their hierarchy are calculated using the mathematics described above. The parts calculated are then written to a database or output. The database can be used for manufacturing simulations or for work content estimations. Figure 5 shows the output of the parts in the software prototype.

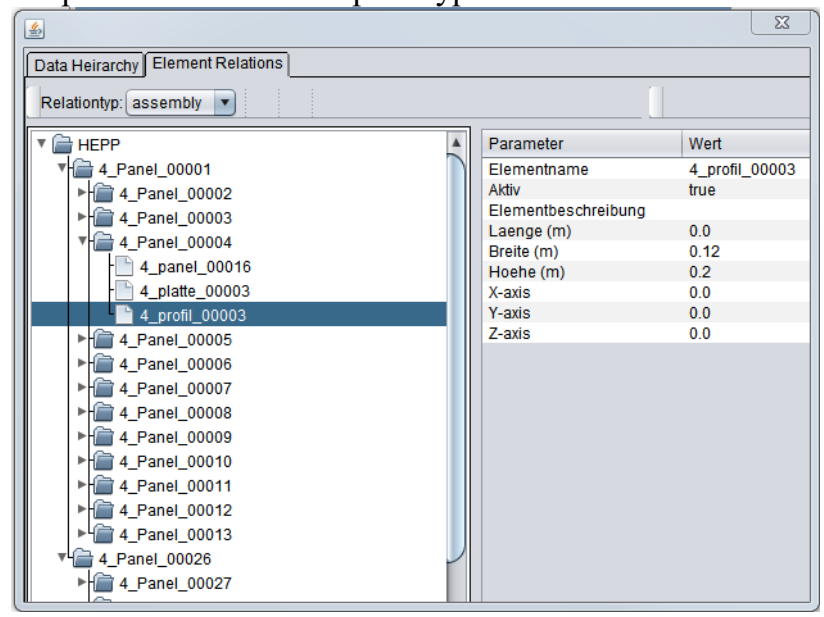

Figure 5. Part view in software prototype

\section{Conclusion}

The above paper explains on how to estimate the work content of a one-of-a-kind product in the early stage of planning. At first a logic on how to describe parts and assemblies in a way, that they can be parameterized, was developed.
In the second step, a software prototype was developed, to validate the logic developed. The software prototype can create parts of an assembly using parametrized templates.

In the future this approach will be tested in reality to allow to compare the estimations done using the tool to the estimations of the current estimation methodology.

\section{Acknowledgments}

The research, described in this paper was conducted in the research project HEPP, in cooperation with German shipyards, funded by the German Federal Ministry of Economic Affairs and Energy (BMWi) due to a decision of the German Bundestag.

\section{References}

1. F. Berner, B. Kochendörfer, R. Schach, Grundlagen der Baubetriebslehre 1. (2007)

2. U. Bracht, S. Wenzel, D. Geckler, Digitale Fabrik, 1. Aufl. (2009)

3. U. Stalleicken, Marktorientierte Produktion komplexer Investitionsgüter - Analysen, Strategien und Konzepte am Beispiel des deutschen Schiffbaus. (2000)

4. R. Lee Storch, C. P. Hammon, H. M. Bunch, R. C. Moore, Ship Production. (1995)

5. H.-P. Wiendahl, Fertigungsregelung: Logistische Beherrschung von Fertigungsabläufen auf Basis des Trichtermodells. (1997)

6. P. J. Fröhlich, Hochbaukosten-FlächenRauminhalte: DIN 276-DIN 277-DIN 18960; Kommentar und Erläuterungen. (2008)

7. B. Ebert, Technische Projekte, 1. Aufl. (2002)

8. C. Lühe, Modulare Kostenschätzung als Unterstützung der Anlagenplanung für die Angebots-und frühe Basic Engineering Phase. (2013)

9. P. Römisch, M. Weiß, Projektierungspraxis Verarbeitungsanlagen. (2014)

10. H. Lödding, A. Friedewald, L. Wagner, R. Wandt, Generisches Daten- und Modellmanagement für die schiffbauliche Produktionssimulation. (2011) 